\title{
Fatigability and blood flow in the rat gastrocnemius- plantaris-soleus after hindlimb suspension
}

\author{
K. S. MCDONALD, M. D. DELP, AND R. H. FITTS \\ Biology Department/Biological and Biomedical Research Institute, Marquette University, Milwaukee, \\ Wisconsin 53233; and Exercise Biochemistry Laboratory, University of Georgia, Athens, Georgia 30602
}

\begin{abstract}
McDonald, K. S., M. D. DelP, AND R. H. FitTs. Fatigability and blood flow in the rat gastrocnemius-plantaris-soleus after hindlimb suspension. J. Appl. Physiol. 73(3): 1135-1140, 1992. -The purpose of this study was to test the hypothesis that hindlimb suspension increases the fatigability of the soleus during intense contractile activity and determine whether the increased fatigue is associated with a reduced muscle blood flow. Cage-control (C) and 15-day hindlimb-suspended (HS) rats were anesthetized, and either the gastrocnemius-plantaris-soleus (G-P-S) muscle group or the soleus was stimulated $(100 \mathrm{~Hz}, 100-\mathrm{ms}$ trains at $120 / \mathrm{min})$ for $10 \mathrm{~min}$ in situ. In the G-P-S preparation, blood flow was measured with radiolabeled microspheres before and at 2 and 10 min of contractile activity. The G-P-S fatigued markedly at this stimulation frequency, and the differences between $\mathrm{C}$ and $\mathrm{HS}$ animals were not significant until the 9 th min of contractile activity. In contrast, the stimulation resulted in faster rates and significantly larger amounts of fatigue in the soleus from HS than from $\mathrm{C}$ animals. The atrophied soleus showed significant differences by $1 \mathrm{~min}$ of stimulation ( $\mathrm{C}=70 \pm 1 \%$ vs. $\mathrm{HS}=57 \pm 2 \%$ of peak train force) and remained different at $10 \mathrm{~min}(\mathrm{C}=64 \pm 4 \%$ vs. $\mathrm{HS}=45 \pm$ $2 \%$ peak train force). Relative blood flow to the soleus was similar between groups before and during contractile activity (rest: $\mathrm{C}=20 \pm 3$ vs. $\mathrm{HS}=12 \pm 3 ; 2 \mathrm{~min}: \mathrm{C}=128 \pm 6$ vs. $\mathrm{HS}=118 \pm 4$; $10 \mathrm{~min}: \mathrm{C}=123 \pm 11$ vs. $\left.\mathrm{HS}=105 \pm 11 \mathrm{ml} \cdot \mathrm{min}^{-1} \cdot 100 \mathrm{~g}^{-1}\right)$. In conclusion, these results established that 15 days of HS increased the fatigability of the soleus, but the effect was not caused by a reduced muscle blood flow.
\end{abstract}

muscle fatigue; muscle atrophy

THE BIOCHEMICAL AND PHYSIOLOGICAL properties of limb skeletal muscle have been shown to adapt to a variety of experimental conditions $(9,13,18)$. Among these is the microgravity encountered with spaceflight $(18,27)$. Studies on skeletal muscles from rats orbited in COSMOS biosatellites and Spacelab 3 reveal multifaceted deterioration, involving muscle fiber atrophy, degeneration of motor innervation, muscle fiber segmental necrosis and central-core lesions, and disruption of the microvasculature $(18,27)$. These changes suggest a spaceflight-induced reduction in muscle work capacity. To study the cellular and molecular mechanisms responsible for these changes, numerous models have been used to mimic the hypokinesia (reduced number of contractions) and hypodynamia (reduced force of contractions) associated with weightlessness (23). One frequently studied model is hindlimb suspension (HS), which has been observed to produce atrophy, central corelike lesions (in $30 \%$ of so- leus fibers), alterations in the enzymatic and contractile properties, and a reduced peak power in individual rat soleus muscle fibers $(6,7,9,10,20,28,29)$.

Fell et al. (6) and Winiarski et al. (29) reported 1 and 4 wk of HS to have no effect on the fatigability of the soleus muscle. However, the train stimulation frequency used by Fell et al. (45/min) and Winiarski et al. (60/min) elicited only minimal fatigue and, thus, may not have been intense enough to uncover differences in the fatigue patterns of control and experimental groups. Witzmann et al. (30) employed a higher stimulation frequency (110 trains $/ \mathrm{min}$ ) and observed greater fatigability of the rat soleus after 42 days of hindlimb immobilization (HI) compared with the control. The effect of high-frequency stimulation on the fatigability of the soleus after HS has not been characterized.

HI has been shown to increase the extent of glycogen and ATP decline and yield a higher lactate content in the soleus during contractile activity $(30)$. Additionally, the glucose uptake capacity and muscle fiber glycogen content were increased in the soleus after HS $(11,12)$, whereas the specific activity of phosphofructokinase and lactate dehydrogenase of single soleus fibers was elevated after 4 wk of $\mathrm{HI}$ and $\mathrm{HS}$ (7). These findings suggest that both models induce a shift in the atrophied soleus toward an increased reliance on glycogen metabolism. This shift occurs despite an increase in the oxidative enzyme capacity of single fibers isolated from the soleus after both $\mathrm{HI}$ and $\mathrm{HS}$ (7). An increased fatigability and dependence on glycogen metabolism in the atrophied soleus despite higher concentrations of aerobic enzymes could be explained by a reduced tissue blood flow. Consequently, the purpose of this study was to test the hypothesis that HS increases the fatigability of the soleus during intense contractile activity and that the increased fatigue is associated with a reduced muscle blood flow.

\section{MATERIALS AND METHODS}

Animal care and suspension procedure. Male SpragueDawley rats (250-275 g) were obtained from Sasco (Madison, WI) and randomly assigned to either the HS or cage-control group. The hindlimbs of the HS animals were elevated for 15 days with use of a harness attached to the proximal two-thirds of the tail, as previously described in detail (9). The height of suspension was adjusted to prevent the hindlimbs from contacting supportive surfaces. The forelimbs maintained contact with a 
grid floor, which allowed the animals to move about to obtain food and water. The HS animals were fed Purina rat chow and water ad libitum, whereas the control rats were pair fed to maintain weights similar to those of the HS group. Both groups were housed at $23^{\circ} \mathrm{C}$ with a 12:12-h light-dark cycle.

Surgical procedures. After 15 days, the HS and control rats were anesthetized with pentobarbital sodium (50 $\mathrm{mg} / \mathrm{kg}$ body wt ip). A Silastic catheter $(0.6 \mathrm{~mm} \mathrm{ID}, 1.0$ $\mathrm{mm}$ OD) was surgically implanted in the ascending aorta via the right carotid artery, as previously described (16). This catheter was subsequently used for recording arterial pressure and the infusion of radiolabeled microspheres for blood flow measurements. A second polyurethane catheter $(0.36 \mathrm{~mm} \mathrm{ID,} 0.84 \mathrm{~mm}$ OD) was inserted $\sim 4 \mathrm{~cm}$ into the tail caudal artery, as described by Chiueh and Kopin (3) with several modifications (4), to withdraw blood for the blood flow measurements.

Muscle preparation and stimulation. After catheter implantation the left gastrocnemius-plantaris-soleus (G-PS) muscle group was prepared for in situ stimulation. The muscle group was exposed and dissected free of surrounding tissue, with its blood and nerve supply left intact. Silk thread (4-0) was secured to the distal end of the Achilles tendon, and a small loop was tied for subsequent attachment to the force transducer. The rat was placed on a fixed platform within a $50 \times 50 \times 90-\mathrm{cm}$ Plexiglas chamber maintained at $35^{\circ} \mathrm{C}$, and the hindlimb was secured as previously described (30). The leg was immobilized at the ankle by a U-bolt fastened to the base of the platform. The skin that had been dissected free from the leg was pulled over a flange on each side of the platform and secured by stainless steel wound clips. The muscle and nerve preparation was kept moist with a gauze pad saturated with rat Ringer solution (in $\mathrm{mM}: 137$ $\mathrm{NaCl}, 4 \mathrm{KCl}, 1 \mathrm{MgCl}_{2}, 1 \mathrm{KH}_{2} \mathrm{PO}_{4}, 12 \mathrm{NaHCO}_{3}$, and 2 $\mathrm{CaCl}_{2}$ ). In a second group of $\mathrm{HS}$ and control animals the soleus, composed primarily of slow-twitch type I fibers (2), was freed of surrounding tissues, with the blood and nerve supply of the G-P-S muscle group remaining intact. The isolation of the soleus involved dividing the gastrocnemius into its medial and lateral heads and carefully pulling back the two heads of the gastrocnemius and the plantaris such that their contribution to soleus force production was minimal. A silk thread loop (4-0) was tied to the distal tendon, the rat was transferred to the Plexiglas chamber, and the leg was fixed to the support, as described above.

Before the contractile properties were measured, the preparation was allowed to thermoequilibrate for $20 \mathrm{~min}$. Muscle temperature for the G-P-S and soleus was estimated by inserting a needle thermistor into the biceps femoris and lateral head of the gastrocnemius, respectively. Core temperature was monitored via a rectal probe. A multichannel telethermometer (Yellow Springs Instruments) displayed the inputs of both probes. The muscle and body temperatures averaged $35.2 \pm 0.2$ and $37.5 \pm 0.1^{\circ} \mathrm{C}$, respectively.

The muscles were stimulated indirectly through the distal portion of the cut sciatic nerve, which was isolated in the gluteal region and drawn into a suction-stimulating electrode. Each muscle preparation was adjusted to its optimal length at which maximal twitch and tetanic force (newtons) was achieved. Twitch contractions were elicited by supramaximal $(1.5-\mathrm{V})$ square-wave pulses of $0.1-\mathrm{ms}$ duration produced by a Grass $\mathrm{S} 48$ stimulator. Tetanic contractions were generated by supramaximal stimulation (2-s duration) at $100 \mathrm{~Hz}$. During the isometric contractions, the force transducer (model FT10C, Grass Instruments) output was amplified and displayed on a pin recorder (model 735-1320, Narco Physiograph).

Fatigue studies. Isometric train force $\left(\mathrm{P}_{\mathrm{tr}}\right)$ of the G-P-S or soleus was recorded at $0,0.5,1,1.5,2,3,4,5,6,7,8,9$, and $10 \mathrm{~min}$ of contractile activity. The muscles were stimulated with $100-\mathrm{ms}$ trains of $100 \mathrm{~Hz}$ at a train rate of $120 / \mathrm{min}$. The train duration was selected to obtain high but not peak tetanic force, because prolonged tetanic contraction might impede blood flow and prevent detection of flow differences between control and HS animals. This stimulation protocol has previously been shown to elicit fatigue and high blood flow in the G-P-S muscle group (17). Muscle blood flow was measured before contractile activity (resting flow) and during the 2nd and 10th min of contraction. Blood flow measurements were made only in the G-P-S preparation.

Blood flow measurements. Radiolabeled $\left({ }^{85} \mathrm{Sr},{ }^{113} \mathrm{Sn}\right.$, and ${ }^{153} \mathrm{Gd}$ ) microspheres (New England Nuclear) with a $15-\mu \mathrm{m}$ diameter were used for blood flow measurements, as previously described (16). The microspheres were suspended in $0.9 \%$ physiological saline containing $<0.01 \%$ Tween 80, mixed for $10 \mathrm{~min}$ in a Mettler Electronics ultrasonicator, and vortexed for $1 \mathrm{~min}$ before infusion. A $0.2 \mathrm{ml}$ aliquot containing 500,000 spheres was infused into the aorta and the catheter flushed with warm saline $\left(37^{\circ} \mathrm{C}\right)$. The infusion process required $\sim 30 \mathrm{~s}$ and thus was begun $15 \mathrm{~s}$ before each measurement period. Radioactivity of the samples was measured with a gamma counter (Packard Auto Gamma 5780), and flows were computed (IBM-PC computer) from counts per minute and tissue wet weights. To ensure that microsphereblood mixing was adequate, flows to right and left kidneys were compared for each animal. Mixing was considered sufficient if kidney flows were within $15 \%$ of each other.

For the blood flow measurements, the entire soleus and plantaris muscles were assayed, whereas the gastrocnemius was divided into white, red, and mixed portions. The white and red portions were obtained by pooling samples obtained from the superficial and deep regions of both heads of the gastrocnemius, respectively. The mixed portion consisted of the remaining gastrocnemius tissue.

Central hemodynamic measurements. Mean arterial pressure and heart rate were recorded from the carotid catheter just before each microsphere infusion with use of a pressure transducer (model RP-1500, Narco BioSystems) and recorder (model 735-1320, Narco physiograph). Only mean arterial pressure was reported because of the damping effect of the catheters. Heart rates were determined from the pulsatile pressure recordings.

Data analysis. Exponential curves of the form of $y_{t}=$ $A e^{-t}+C$, where $y_{t}$ is developed force at time $t, A$ is a constant of the slope function, and $C$ is the asymptote of developed force, were fit to the time patterns of fatigue, 
TABLE 1. Tissue weights

\begin{tabular}{lcccccc}
\hline \hline & \multicolumn{2}{c}{ Tissue Wt, $\mathrm{mg}$} & & \multicolumn{2}{c}{ Tissue Wt/Body Wt, mg/g } \\
\cline { 2 - 3 } \multicolumn{1}{c}{ Tissue } & $\mathrm{C}$ & $\mathrm{HS}$ & & $\mathrm{C}$ & HS \\
\hline Soleus & $185 \pm 5$ & $\mathbf{1 0 6} \pm 6^{*}$ & & $0.52 \pm 0.01$ & $0.32 \pm 0.02^{*}$ \\
Plantaris & $\mathbf{4 4 5 \pm 1 4}$ & $355 \pm 15^{*}$ & & $1.25 \pm 0.03$ & $1.07 \pm 0.03^{*}$ \\
Gastrocnemius & $2,147 \pm 55$ & $1,661 \pm 68^{*}$ & & $6.05 \pm 0.19$ & $5.03 \pm 0.12^{*}$ \\
Left kidney & $1,166 \pm 45$ & $1,270 \pm 40$ & & $3.29 \pm 0.17$ & $3.85 \pm 0.07^{*}$ \\
Right kidney & $1,168 \pm 44$ & $1,303 \pm 46$ & & $3.31 \pm 0.15$ & $4.00 \pm 0.10^{*}$ \\
\hline
\end{tabular}

Values are means $\pm \mathrm{SE}$ of 8 observations. $\mathrm{C}$, control group; HS, 15-day hindlimb-suspended group. ${ }^{*} \mathrm{HS}$ significantly different from $\mathrm{C}$, $P<0.05$.

and the curves were analyzed by $t$ test statistics. A twoway analysis of variance was used to compare heart rates, mean arterial pressures, and blood flows within tissues between control and HS groups and across rest and 2 and $10 \mathrm{~min}$ of contractile activity. When significant differences occurred, a Student's unpaired two-tailed $t$ test was used as the post hoc test to determine statistical significance between groups. The 0.05 level of probability was set for statistical significance. Reported values represent means $\pm \mathrm{SE}$.

\section{RESULTS}

Tissue weights. Significant reductions in the soleus, plantaris, and gastrocnemius muscle wet weights occurred as a result of 15 days of HS (Table 1). Consistent with observations by others $(6,9,10)$, the soleus muscle demonstrated the greatest atrophy after HS. The absolute soleus muscle weight was $43 \%$ lower than control after HS ( $185 \pm 5$ vs. $106 \pm 6$ ), whereas the soleus muscle-to-body weight ratio was $38 \%$ lower than controls $(0.52 \pm 0.02$ vs. $0.32 \pm 0.02)$. The muscle weight-to-body weight ratios of the plantaris and gastrocnemius after HS were 14 and $17 \%$ lower than control values, respectively. The elevated kidney-to-body weight ratio of HS animals can likely be attributed to the selective loss of muscle mass.

Fatigue studies. The fatigue patterns of both the soleus and G-P-S are shown in Fig. 1. The initial $P_{t r}$ of the G-P$\mathrm{S}$ muscle group was significantly lower after 15 days of $\mathrm{HS}(\mathrm{C}=18 \pm 1$ vs. $\mathrm{HS}=15 \pm 1 \mathrm{~N})$. Similarly, the initial soleus $\mathrm{P}_{\mathrm{tr}}$ for control $(2.02 \pm 0.09 \mathrm{~N})$ and $\mathrm{HS}(1.10 \pm 0.07$ $\mathrm{N})$ animals was significantly different. However, when expressed per gram tissue, the soleus $P_{t r}$ was not significantly different between groups (control $=11.13 \pm 0.34$ vs. $\mathrm{HS}=11.29 \pm 0.74 \mathrm{mN} / \mathrm{mg}$ tissue). The initial $\mathrm{P}_{\mathrm{tr}}$ values averaged $71 \pm 1$ and $73 \pm 2 \%$ of peak tetanic tension produced by the soleus from control and 15-day HS animals, respectively. All the contractile activity curves are characterized by a biphasic response, with an initial (1-2 min) rapid fall in force followed by a steady-state phase in which force is maintained or falls slightly. During the 10-min train stimulation period the soleus of the control and HS rats fatigued an average of 34 and $52 \%$ from their initial train force, respectively. Except for the first $30 \mathrm{~s}$, the percent decline in tension was significantly greater in the HS group throughout the stimulation protocol. Exponential curves of the form $y_{t}=A e^{-t}+C$ were fit to the individual fatigue patterns. The equation de- scribing the soleus fatigue pattern in the control animals was $y_{t}=34 e^{-t}+66$ and in the HS rats was $y_{t}=52 e^{-t}+48$.

The equations describing the G-P-S muscle group fatigue patterns were $y_{t}=69 e^{-t}+31$ for controls and $y_{t}=$ $73 e^{-t}+27$ for HS animals. The curves were significantly different between groups; however, the percent decline in force was not significantly greater in the HS group until 9 min of activation.

Blood flow measurements. The absolute blood flows $(\mathrm{ml} / \mathrm{min})$ and blood flows expressed per $100 \mathrm{~g}$ tissue weight $\left(\mathrm{ml} \cdot \mathrm{min}^{-1} \cdot 100 \mathrm{~g}^{-1}\right)$ during rest and isometric contractile activity are presented in Table 2 . The absolute blood flow to the soleus was significantly lower in the HS than in the control animals at all three time points. However, when normalized to muscle weight, soleus blood flow was not different between groups at rest or during contractile activity (Table 2).

During contractile activity both the absolute and normalized blood flow to the plantaris and white gastrocnemius were significantly lower in the HS animals (Table 2 ). The absolute flows of the red gastrocnemius $(2 \mathrm{~min})$ and mixed gastrocnemius ( 2 and $10 \mathrm{~min}$ ) were significantly lower in the HS than in the control animals (Table 2). However, when expressed per tissue weight, the blood flow to these tissues was not different between groups.

Heart rate and blood pressure. Mean heart rate and arterial blood pressure values for control and HS animals during rest and contractile activity are presented in Table 3. Both heart rate and mean arterial pressure remained stable during contractile activity and were not different between groups.

\section{DISCUSSION}

The purpose of this study was to test the hypothesis that 15 days of HS reduces the blood flow and increases the fatigability of the slow-twitch soleus muscle during intense contractile activity. The results indicate that after 15 days of $\mathrm{HS}$ the soleus had a reduced resistance to fatigue; however, soleus blood flow per $100 \mathrm{~g}$ muscle was

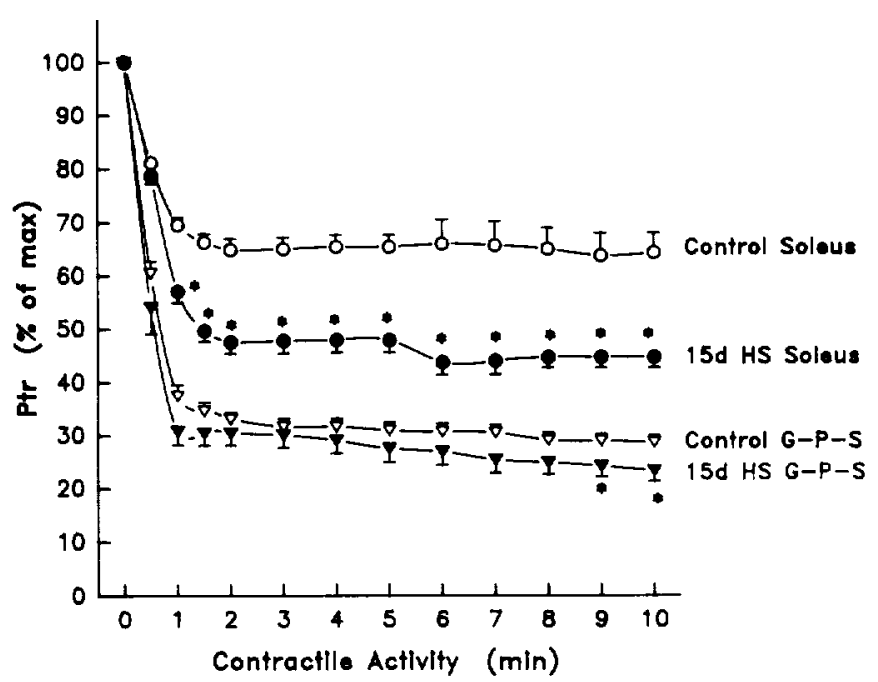

FIG. 1. Effect of 15 days of hindlimb suspension (HS) on peak train force $\left(P_{t r}\right)$ relative to initial values during contractile activity in soleus and gastrocnemius-plantaris-soleus (G-P-S) muscle group. Each point is mean $\pm \mathrm{SE}$ for 8 observations. ${ }^{*}$ Control vs. 15 -day HS, $P<0.05$. 
TABLE 2. Blood flows in tissues and organs during rest and contractile activity

\begin{tabular}{|c|c|c|c|c|}
\hline \multirow[b]{3}{*}{ Tissue } & \multicolumn{4}{|c|}{ Blood Flow } \\
\hline & \multicolumn{2}{|c|}{$\mathrm{ml} \cdot \min ^{-1} \cdot 10^{-2}$} & \multicolumn{2}{|c|}{$\mathrm{ml} \cdot \min ^{-1} \cdot 100 \mathrm{~g}^{-1}$} \\
\hline & $\mathrm{C}$ & HS & $\mathrm{C}$ & HS \\
\hline \multicolumn{5}{|l|}{ Soleus } \\
\hline Rest & $3.7 \pm 0.5$ & $1.3 \pm 0.3^{*}$ & $20 \pm 3$ & $12 \pm 3$ \\
\hline $2 \min$ & $23.6 \pm 1.2$ & $12.5 \pm 0.8^{*}$ & $128 \pm 6$ & $118 \pm 4$ \\
\hline $10 \mathrm{~min}$ & $22.6 \pm 1.9$ & $10.8 \pm 0.9^{*}$ & $123 \pm 11$ & $105 \pm 11$ \\
\hline \multicolumn{5}{|l|}{ Plantaris } \\
\hline Rest & $2.9 \pm 1.0$ & $2.2 \pm 0.6$ & $6 \pm 2$ & $7 \pm 2$ \\
\hline $2 \min$ & $66.0 \pm 10.0$ & $33.0 \pm 3.8^{*}$ & $146 \pm 18$ & $94 \pm 11^{*}$ \\
\hline $10 \mathrm{~min}$ & $74.3 \pm 12.0$ & $33.8 \pm 5.5^{*}$ & $165 \pm 22$ & $97 \pm 17^{*}$ \\
\hline \multicolumn{5}{|c|}{ Red gastrocnemius } \\
\hline Rest & $2.2 \pm 0.8$ & $1.5 \pm 0.4$ & $23 \pm 9$ & $18 \pm 6$ \\
\hline $2 \min$ & $26.6 \pm 2.5$ & $19.5 \pm 1.7^{*}$ & $253 \pm 26$ & $232 \pm 16$ \\
\hline $10 \mathrm{~min}$ & $27.0 \pm 3.7$ & $20.0 \pm 2.6$ & $273 \pm 41$ & $236 \pm 24$ \\
\hline \multicolumn{5}{|c|}{ White gastrocnemius } \\
\hline Rest & $1.7 \pm 0.3$ & $1.5 \pm 0.4$ & $11 \pm 2$ & $12 \pm 4$ \\
\hline $2 \min$ & $10.3 \pm 2.0$ & $4.1 \pm 0.8^{*}$ & $65 \pm 12$ & $32 \pm 6^{*}$ \\
\hline \multirow{2}{*}{\multicolumn{5}{|c|}{ Mixed gastrocnemius }} \\
\hline & & & & \\
\hline Rest & $22.2 \pm 5.0$ & $20.6 \pm 6.8$ & $12 \pm 3$ & $15 \pm 5$ \\
\hline $2 \min$ & $271.0 \pm 33.0$ & $161.0 \pm 20.0^{*}$ & $144 \pm 17$ & $113 \pm 15$ \\
\hline $10 \mathrm{~min}$ & $312.0 \pm 32.0$ & $168.0 \pm 27.0^{*}$ & $168 \pm 18$ & $120 \pm 20$ \\
\hline \multicolumn{5}{|l|}{ Left kidney } \\
\hline Rest & $618.0 \pm 56.0$ & $714.0 \pm 96.0$ & $526 \pm 34$ & $559 \pm 70$ \\
\hline $2 \min$ & $768.0 \pm 126.0$ & $816.0 \pm 100.0$ & $645 \pm 86$ & $638 \pm 67$ \\
\hline $10 \mathrm{~min}$ & $686.0 \pm 95.0$ & $806.0 \pm 83.0$ & $611 \pm 88$ & $635 \pm 61$ \\
\hline \multicolumn{5}{|l|}{ Right kidney } \\
\hline Rest & $655.0 \pm 53.0$ & $731.0 \pm 98.0$ & $554 \pm 30$ & $555 \pm 65$ \\
\hline $2 \min$ & $809.0 \pm 135.0$ & $836.0 \pm 11.0$ & $673 \pm 91$ & $634 \pm 65$ \\
\hline $10 \mathrm{~min}$ & $714.0 \pm 108.0$ & $868.0 \pm 81.0$ & $629 \pm 96$ & $663 \pm 50$ \\
\hline
\end{tabular}

Values are means $\pm \mathrm{SE}$ of 8 observations. ${ }^{*} \mathrm{HS}$ significantly different from $\mathrm{C}, P<0.05$.

not altered. These findings suggest that the increased fatigability of the atrophied soleus cannot be accounted for by a reduced blood flow to soleus muscle cells.

The stimulation protocol selected was known to elicit high muscle blood flow and significant fatigue in G-P-S muscles of control animals. Fitts and Holloszy (8) and Witzmann et al. (30) demonstrated that a train stimulation frequency of $110 / \mathrm{min}$ elicited a significant decline $(\sim 30 \%)$ in the $P_{t r}$ of the soleus after 30 min of contractile activity. Mackie and Terjung (17) showed that a train stimulation frequency of $120 / \mathrm{min}$ caused both significant fatigue of the G-P-S muscle group and high blood flow to these muscles. The muscle blood flow at rest and during contractile activity reported in this study for the soleus and red and white gastrocnemius (Table 2) was essentially identical to that observed by Mackie and Terjung in the anesthetized rat hindlimb (see Ref. 17, Fig. 5).

The resting soleus blood flow in the control group was lower than that in awake standing rats $(20 \pm 3$ vs. $36 \pm 5$ $\left.\mathrm{ml} \cdot \min ^{-1} \cdot 100 \mathrm{~g}^{-1}\right)$ but higher than that measured during acute $(5 \mathrm{~min}) \mathrm{HS}$ in conscious animals $(20 \pm 3 \mathrm{vs} .8 \pm$ $1 \mathrm{ml} \cdot \mathrm{min}^{-1} \cdot 100 \mathrm{~g}^{-1}$ ) (19). The somewhat lower muscle blood flow in the anesthetized rat than in the awake standing rat was not surprising in that the hindlimbs of the anesthetized rat were unloaded. After HS, the resting soleus blood flow in the anesthetized rat was reduced by $40 \%$ compared with the control group. Although not statistically significant, this change was consistent with the observation that HS significantly reduced the soleus blood flow in the awake standing rat (19). The hindlimb muscle blood flows elicited with electrical stimulation in this study and that of Mackie and Terjung (17) were higher than those observed during treadmill walking at $15 \mathrm{~m} / \mathrm{min}$ (19) but lower than those obtained during heavy treadmill exercise $(1,15)$. Mackie and Terjung found hindlimb muscle blood flow to decrease with stimulation frequencies $<120 / \mathrm{min}$. Consequently, it seems reasonable to suggest that the hindlimb blood flows elicited by contractile activity in this study were the peak flows obtainable during in situ electrical stimulation. Although 15 days of HS did not affect soleus blood flow, the flow to the white gastrocnemius was significantly depressed during contractile activity. This finding differs from the in vivo conditions in which flow to the white gastrocnemius (and other fast-twitch glycolytic muscle) was increased after HS both at rest and during treadmill walking (19). We hypothesized that this increased flow was mediated by a downregulation in sympathetic-mediated vasoconstriction (19). The reduced flow in the white gastrocnemius during intense in situ contractile activity suggests greater vasoconstriction in this muscle after HS. The possibility exists that metabolic factors (such as an increased $\mathrm{H}^{+}$) triggered a chemoreflex, causing vasoconstriction (22). Thus this work and our previous study (19) suggest that HS modifies the sympathetic-mediated vascular tone in both active and inactive white fast-twitch muscle.

After 15 days of HS the soleus muscle was significantly less resistant to fatigue during $10 \mathrm{~min}$ of electrical stimulation. In contrast, Fell et al. (6) found 1 wk of whole body suspension to yield no differences in fatigue between atrophied and control soleus. Additionally, Winiarski et al. (29) found 4 wk of HS not to affect the fatigue index (ratio of force developed after 2 min of stimulation to maximum force) in the soleus. These differences are likely attributed to the variations in stimulation protocols. The higher train stimulation frequency used in the present study $(120 / \mathrm{min})$ revealed fatigue differences between control and atrophied soleus not observed with the lower frequencies of 45 and 60 trains $/ \mathrm{min}$ employed by Fell et al. and Winiarski et al., respectively. Other possibilities for the discrepancy in soleus fatigability could include 1) duration of $\mathrm{HS}, 2$ ) age and sex of the rats, and 3) different experimental techniques.

Several possibilities could explain the increased fatigability of the soleus after HS despite no difference in blood flow. Kandarian et al. (14) recently observed a twofold increase in the relative interstitial volume of the soleus after $4 \mathrm{wk}$ of $\mathrm{HS}$, a change that could increase the diffu-

TABLE 3. Heart rate and blood pressure

\begin{tabular}{llllll}
\hline \hline & \multicolumn{2}{c}{ Heart Rate, beats/min } & & \multicolumn{2}{c}{$\begin{array}{c}\text { Mean Arterial Bload } \\
\text { Pressure, mm Hg }\end{array}$} \\
\cline { 2 - 3 } \cline { 5 - 7 } Condition & $\mathrm{C}$ & HS & & $\mathrm{C}$ & HS \\
\hline Rest & $379 \pm 14(8)$ & $398 \pm 14(8)$ & & $125 \pm 6(8)$ & $121 \pm 5(8)$ \\
$2 \mathrm{~min}$ & $380 \pm 15(8)$ & $399 \pm 9(8)$ & & $124 \pm 9(8)$ & $114 \pm 5(8)$ \\
$\mathbf{1 0} \mathrm{min}$ & $389 \pm 13(7)$ & $411 \pm 11(8)$ & & $126 \pm 5(7)$ & $117 \pm 7(8)$ \\
\hline
\end{tabular}

Values are means $\pm S E$ for no. of observations in parentheses. 
sion distance for oxygen exchange between the capillary and muscle cell. However, extracellular volume was unaltered immediately after 2 wh of HS, and interstitial edema did not develop until $>12 \mathrm{~h}$ of hindlimb reloading (D. A. Riley, personal communication). Furthermore, Desplanches et al. (5) observed that capillary density was increased after $\mathrm{HS}$, and this plus the smaller fiber diameter associated with HS should increase the surface-tovolume ratio and thus reduce the diffusion distances. A second possibility is an HS-induced decrease in responsiveness to sympathetic activation. HS may cause a chronic stimulation of sympathetic drive, yielding a downregulation of adrenergic receptors. In fact, Overton and Tipton (25) found 9 days of HS to blunt the mesenteric artery pressor response to exogenous sympathomimetic agents. Additionally, we recently found 15 days of $\mathrm{HS}$ to attenuate the redistribution of visceral blood flow normally observed during exercise (19). These findings could be caused by a reduced number and/or sensitivity of $\alpha$-receptors after HS. The consequence of this may be a decreased cellular response to sympathetic drive, which could inhibit the mobilization of free fatty acids (FFA), thus reducing their delivery, uptake, and oxidation by skeletal muscle. The working muscle would then be more dependent on the oxidation of carbohydrates for energy production.

A reduced liberation of FFA and their subsequent delivery and oxidation by the working muscle may have contributed to an increased fatigue and glycogen usage during the second steady-state portion of the stimulation period. However, the initial rapid phase of fatigue most likely occurred before significant amounts of FFA could be mobilized from adipose cells, delivered, and oxidized in working muscle. Thus, additional factors must be responsible for the augmented early phase of fatigue in the atrophied soleus.

The more rapid fall in peak train force in the atrophied soleus may involve neural and/or excitation-contraction coupling processes. Fitts and Holloszy (8) observed a similar contractile response of the soleus to both direct and indirect stimulation after fatigue. This suggests that the fatigue induced in the control soleus was not due to alterations in neuromuscular transmission. However, the effect of HS on neuromuscular transmission is unknown. Additionally, the atrophied muscle may be less effective at coupling excitation and contraction. The effects of unweighting on the excitation-contraction coupling process (e.g., T-tubular charge movement and sarcoplasmic reticulum $\mathrm{Ca}^{2+}$ release) have not been investigated.

Another possibility for the increase in hindlimb muscle fatigability despite no difference in blood flow may be a lower blood and/or muscle oxygen-carrying capacity after HS. A reduction in erythrocyte concentration has been reported after HS (26). To our knowledge, there are no studies that have measured the effect of weightlessness on myoglobin concentration.

Finally, the greater fatigability of the soleus after HS could be in part mediated by an increased type IIa-totype I fiber ratio (5). During contractile activity, predominantly fast-twitch limb muscles are known to fatigue more rapidly and generate higher lactates, $\mathrm{H}^{+}$, and inorganic phosphate (30). Both of the latter two ions have been shown to reduce peak force $(21,24)$.
Even though the isolated soleus exhibited greater fatigue after HS, the fatigue pattern of the G-P-S muscle group was not different between groups until the 9 th min of activation. The fiber mass of the G-P-S contains $\sim 65 \%$ fast-twitch glycolytic fibers (2). Therefore the GP-S fatigue pattern likely reflects the response of this fiber type, a fiber known to depend primarily on anaerobic metabolism and thus unlikely to be affected by changes in substrate or oxygen delivery. Why the fatigue pattern of the G-P-S differed between groups late in the bout of activation is unknown. One possibility is that by the 9 th min of activation the force output of the gastrocnemius and plantaris may have sufficiently declined such that the difference in soleus force becomes significant.

The observation that the fatigue pattern of the G-P-S was not different between groups (except in the final minute of activation) despite significantly lower blood flow $\left(\mathrm{ml} \cdot \mathrm{min}^{-1} \cdot 100 \mathrm{~g}^{-1}\right)$ to the plantaris and white gastrocnemius suggests that the maintenance of force output was not limited by blood flow to the G-P-S in either group.

In conclusion, after 15 days of $\mathrm{HS}$, the soleus muscle exhibited marked atrophy and fatigued to a greater extent than control soleus. However, despite the increased fatigability of the atrophied soleus, the blood flow per gram muscle was similar to control values. This result suggests that the greater fatigability of the atrophied soleus was not caused by a reduced blood flow.

The authors are grateful to Barbara DeNoyer for help in the preparation of the manuscript, Dr. Robert B. Armstrong for advice on the design of the blood flow experiments, and Dr. Naveen Bansel for statistical analysis consultation.

This study was supported by National Aeronautics and Space Administration Grant NAG 2-212.

Address for reprint requests: R. H. Fitts, Biology Dept., Marquette University, Milwaukee, WI 53233.

Received 19 September 1991; accepted in final form 14 April 1992.

\section{REFERENCES}

1. ARmstrong, R. B., AND M. H. Laughlin. Rat muscle blood flows during high-speed locomotion. J. Appl. Physiol. 59: 1322-1328, 1985.

2. Armstrong, R. B., AND R. O. Phelps. Muscle fiber type composition of the rat hindlimb. Am. J. Anat. 171: 259-272, 1984.

3. ChIUer, C. C., AND I. J. KopIN. Hyperresponsivity in spontaneously hypertensive rat to indirect measurement of blood pressure. Am. J. Physiol. 234 (Heart Circ. Physiol. 3): H690-H695, 1978.

4. DElP, M. D., AND R. B. ARMSTrong. Blood flow in normal and denervated muscle during exercise in conscious rats. $A m$. J. Physiol. 255 (Heart Circ. Physiol. 24): H1509-H1515, 1988.

5. Desplanches, D., M. H. Mayet, B. Sempore, and R. FlanDRoIS. Structural and functional responses to prolonged hindlimb suspension in rat muscle. J. Appl. Physiol. 63: 558-563, 1987.

6. Fell, R. D., L. B. Gladden, J. M. Steffen, and X. J. Musacchia. Fatigue and contraction of slow and fast muscles in hypokinesia/ hypodynamia rats. $J$. Appl. Physiol. 58: 65-69, 1985.

7. Fitts, R. H., C. J. Brimmer, A. Heywood-Cooksey, and R. J. Timmerman. Single muscle fiber enzyme shifts with hindlimb suspension and immobilization. Am. J. Physiol. 256 (Cell Physiol. 25): C1082-C1091, 1989.

8. FitTs, R. H., AND J. O. Holloszy. Contractile properties of rat soleus muscle: effects of training and fatigue. Am. J. Physiol. 233 (Cell Physiol. 2): C86-C91, 1977

9. Fitts, R. H., J. M. Mftzger, D. A. Riley, and B. R. Unsworth Models of disuse: a comparison of hindlimb suspension and immobilization. J. Appl. Physiol. 60: 1946-1953, 1986.

10. Gardetto, P. R., J. M. Schluter, and R. H. FitTs. Contractile 
function of single muscle fibers after hindlimb suspension. J. Appl. Physiol. 66: 2739-2749, 1989.

11. Henriksen, E. J., K. J. Rodnick, C. E. Mondon, D. E. James, AND J. O. Holloszy. Effect of denervation or unweighting on GLUT-4 protein in rat soleus muscle. J. Appl. Physiol. 70: 2322$2327,1991$.

12. HEYwood-CoOKSEY, A., AND R. H. FrTTS. The effect of hindlimb suspension on the substrate profile of fast and slow skeletal muscle fiber types (Abstract). Med. Sci. Sports Exercise 23: S129, 1991.

13. Holloszy, J. O., AND F. W. Booth. Biochemical adaptations to endurance exercise in muscle. $J$. Appl. Physiol. 56: 831-838, 1976.

14. Kandarian, S. C., R. C. Boushel, AND L. M. Schulte. Elevated interstitial fluid volume in rat soleus muscles by hindlimb unweighting. J. Appl. Physiol. 71: 910-914, 1991.

15. Laughlin, M. H., AND R. B. Armstrong. Muscular blood flow distribution patterns as a function of running speeds in rats. Am. J. Physiol. 243 (Heart Circ. Physiol. 12): H296-H306, 1982.

16. Laughlin, M. H., R. B. Armstrong, J. White, AND K. Rouk. A method for using microspheres to measure muscle blood flow in exercising rats. J. Appl. Physiol. 52: 1629-1635, 1982.

17. MACKIE, B. G., AND R. L. TerJung. Blood flow to different skeletal muscle fiber types during contraction. Am. J. Physiol. 245 (Heart Circ. Physiol. 14): H265-H275, 1983.

18. Martin, T. P., V. R. Edgerton, and R. E. Grindel.and. Influence of spaceflight on rat skeletal muscle. J. Appl. Physiol. 65: 2318-2325, 1988 .

19. McDonald, K. S., M. D. Delp, and R. H. FitTs. Effect of hindlimb unweighting on tissue blood flow in the rat. J. Appl. Physiol. 72: 2210-2218, 1992

20. McDonald, K. S., and R. H. FitTs. Time course of change in single soleus fiber peak shortening velocity, power, and ATPase elicited by hindlimb suspension (Abstract). Med. Sci. Sports Exercise 24: S4, 1992.
21. Metzger, J. M., AND R. L. Moss. Greater hydorogen ion-induced depression of tension and velocity in skinned single fibers of rat fast than slow muscles. J. Physiol. Lond. 393: 727-742, 1987.

22. MrTCHELL, J. H., AND R. F. SCHMIDT. Cardiovascular reflex control by afferent fibers from skeletal muscle receptors. In: Handbook of Physiology. The Cardiovascular System. Peripheral Circulation and Organ Blood Flow. Bethesda, MD: Am. Physiol. Soc., 1983, sect. 2, vol. III, p. 623-658.

23. Musacchia, X. J., J. M. STEFFEn, AND R. D. Fell. Disuse atrophy of skeletal muscle: animal models. Exercise Sport Sci. Rev. 16: 6187, 1988.

24. NoseK, T. M., K. Y. Fender, AND R. E. GoDT. It is diprotonated inorganic phosphate that depresses force in skinned skeletal muscle fibers. Science Wash. DC 236: 191-193, 1987.

25. Overton, J. M., AND C. M. TiPTon. Effect of hindlimb suspension on cardiovascular responses to sympathomimetics and lower body negative pressure. J. Appl. Physiol. 68: 355-362, 1990.

26. Overton, J. M., C. R. Woodman, and C. M. Tipton. Effect of hindlimb suspension on $\dot{V}_{\mathrm{o}_{\text {max }}}$ and regional blood flow responses to exercise. J. Appl. Physiol. 66: 653-659, 1989.

27. Riley, D. A., E. I. Illyina-Kakueva, S. Ellis, J. L. W. Bain, G. R. Slocum, AND F. R. SEdlak. Skeletal muscle fiber, nerve, and blood vessel breakdown in space-flown rats. FASEB $J$. 4: 84-91, 1990.

28. Riley, D. A., G. R. Slocum, J. L. W. Bain, F. R. Sedlak, T. E. SOWA, AND J. W. MELLENDER. Rat hindlimb unloading: soleus histochemistry, ultrastructure, and electromyography. J. Appl. Physiol. 69: 58-66, 1990.

29. Winiarski, A. M., R. R. Roy, E. K. Alford, P. C. Chlang, ANd V.R. EDGERTON. Mechanical properties of rat skeletal muscle after hindlimb suspension. Exp. Neurol. 96: 650-660, 1987.

30. Witzmann, F. A., D. H. Kim, AND R. H. FitTs. Effect of hindlimb immobilization on the fatigability of skeletal muscle. J. Appl. Physiol. 54: 1242-1248, 1983. 